\title{
OBSERVACIONES PERSONALES SOBRE EL SINDROME DE OVARIOS POLIQUISTICOS
}

\author{
Dr. Leonardo Tovar R.* \\ Dr. Herbert Wagner C.**
}

El síndrome de ovarios poliquisticos, fué descrito por primera vez por Stein, quien en 1926 anota como posible causa de esterilidad y de amenorrea, la poliquistosis ovárica bilateral (2-8). En 1936 Stein y Leventhal describieron un síndrome caracterizado (5) por irregularidades menstruales, agrandamiento bilateral de los ovarios con espesamiento de la albugínea, multitud de pequeños quistes en los folículos y masculinización, caracterizada principalmente por hirsutismo. Hay anotaciones anteriores a estas fechas en las cuales se conocía la presencia de ovarios grandes, pálidos, pero no se conocía su etiología endocrina (2-4-8).

El estudio de esta entidad ha traído una serie de hechos en parte contradictorios, ya que se han suscitado diversas teorías en relación con la etiología del síndrome, llegándose a diferenciar estados de simple polimicroquistosis ovárica, y estados de poliquistosis con hipertecosis y virilización (2-8).

Además, paralelamente se han producido avances en el estudio de las glándulas endocrinas que tienen una posible participación en el desarrollo de esta entidad, tales como las glándulas suprarrenales, el ovario y

la hipófisis (1-2). También se han empleado varios tratamientos a base de cortisona, prednisona y anovulatorios; algunos autores reportan éxito y otros fracaso, siendo en la actualidad el tratamiento de elección por ser eficaz en el $100 \%$ de los casos, la resección cuneiforme de los ovarios, preconizada por Stein (1-7) y confirmada por nuestra experiencia. En el presente trabajo tratamos de relatar nuestras experiencias personales encontradas en el estudio de pacientes vistas en las consultas endocrinológica y ginecológica del ICSS.

\section{Material y Métodos}

Se estudian nueve pacientes que presentaban el síndrome de Stein-Leventhal y cuatro pacientes controles.

Estas pacientes fueron vistas en la consulta ginecológica y endocrinológica de los autores del trabajo.

Se llevó a cabo el estudio clínico, el cual se complementó con análisis de 17 cetoesteroides según técnica de Wilson Nathanson y Landan; $17 \mathrm{hi}$ drocorticosteroides según técnica de Porter-Silver; cromatina sexual; dosificación de gonadotropinas hipofisa-

* Endocrinólogo del ICSS.

** Ginecólogo del ICSS. 
rias por el método de Klinfelter; citología vaginal funcional; biopsia de endometrio; estudios radiológicos de Ginecografía y radiografía de Silla Turca y Anatomía Patológica.

\section{Casos Clínicos}

CASO No 1 - Historia No 628968 - G. S. Paciente de 18 años, soltera. Menarquia a los 14 años. Ciclos 28/2. Examen ginecológico: Normal. Hipertricosis marcada. T.A. 110/70. Cromatina sexual negativa en tres análisis diferentes. Se practicó ginecografía la cual reporta la presencia de ovarios poliquísticos bilaterales. 17 cetoesteroides: $15.6 \mathrm{mgm}$. \%. 17 hidrocorticosteroides $5.4 \mathrm{mgm}$. \%. Se practicó resección cuneiforme de ovarios. Dr. Héctor Bernal, y estudio anatomopatológico cuyo resultado fué: "Ovarios poliquísticos foliculares". Además se practicó dosificación de 17 cetoesteroides después de la operación dando la cifra de 12.9 mgm.\%. Se anota que esta paciente presentaba una Triconeurosis acentuada.

CASO No 2 - Historia No 496909 - M. M. Edad 22 años, soltera. Menarquia 13 años. Ciclos: 27/5. Hipertricosis avanzada. Dismenorrea. T.A. 110/70. Examen ginecológico negativo. Cromatina sexual positiva. La Ginecografía reveló ovarios poliquísticos bilaterales. Dosificación de 17 cetoesteroides: $7.5 \%$ mgm. $\%$. 17 hidroxicorticosteroides: $5.4 \mathrm{mgm}$. \%. Se practicó resección cuneiforme de ovarios, Dr. Humberto Correa y estudio anatomopatológico: "Quistes foliculares con proliferación de células tecales internas". Esta paciente presentaba también Triconeurosis. Inicialmente se trató con prednisolona sin resultado.

CASO No 3 - Historia No 670009. B. Q. Edad: 26 años. Soltera. Menarquia a los 15 años. Ciclos 100/8. Examen ginecológico: Normal. Hipertricosis marcada. T.A. normal. Triconeurosis. Cromatina sexual positiva. La Ginecografía mostró ovarios poliquísticos aumentados de tamaño. Dosificaciñn de 17-cetoesteroides $13.5 \mathrm{mgm}$.\% 17 hidrocorticosteroides: 5.4 mgm.\%. Se practicó resección cuneiforme de ovarios, Dr. Alfonso González, y estudio anatomopatológico: "Ovario: Numerosos quistes de tipo folicular... Hiperplasia de células de la teca interna: Estroma fibroso y compacto. Ovarios poliquísticos":

CASO No 4 - Historia No 433406. B. T. Edad: 30 años. Soltera. Menarquia a los 16 años. Ciclos 45/2. Examen ginecológico, normal. Hipertricosis marcada. Acné. T.A. normal. Cromatina sexual positiva. La Ginecografía señala la presencia de ovarios poliquísticos bilaterales.
17 cetoesteroides: 25 mgm.\%. 17 hidrocorticosteroides: 12.5 mgm.\%. Pregnandiol: 5.8 mgm.\%. F. S. H.: positiva con 6.6 U. R; negativo con $19 \mathrm{U}$. Ratón. No se practicó tratamiento quirúrgico. Se hizo tratamiento médico a base de prednisolona con resultados negativos.

CASO No 5 - Historia No 407924. M. R. Edad: 24 años. Soltera. Menarquia a los 15 años. Ciclos: 40/3. Hirsutismo moderado. Dismenorrea severa. T.A. 110/80. Cromatina sexual positiva. La Ginecografía reveló ovarios poliquísticos. Se trató con anovulatorios, sin resultados positivos.

CASO No 6 - Historia No 658464. E. R. Edad: 20 años. Soltera. Menarquia: 11 años. Ciclos: 120/8. Hirsutismo moderado. Dismenorrea severa. T.A. 100/70. La Ginecografía confirmó el diagnóstico. Dosificación de 17 cetoesteroides: $13 \mathrm{mgm}$.\%. No se practicó tratamiento quirúrgico.

CASO No 7 - Historia No 433256. Edad: 24 años. Casada. Antecedentes familiares: Vello en madre y una tía. Antecedentes personales: Amigdalectomía. Pielonefritis. Antecedentes ginecológicos: Menarquia. 16 años. Ciclos: $240 / 3$ Enfermedad actual: Casó a los 22 años. A los 18 años comenzó a notar vello en la cara, lo cual le preocupó y posteriormente comenzó a depilarse, pero el vello siguió aumentando. Tomó Anovlar por algún tiempo pero no lo toleró. Tomó prednisolona $15 \mathrm{mgm}$. diarios por 6 meses y aumentó de peso; después siguió con $10 \mathrm{mgm}$. diarios por dos meses, y finalmente 5 mgm. y $2.5 \mathrm{mgm}$. diarios. - Examen físico: Peso: $51 \mathrm{kgm}$. Talla: $1.62 \mathrm{~m}$. T.A. 120/70. Pulso: $76 / \mathrm{m}$. Facies: Vello en el labio superior y mentón. Cuello: normal. Tórax: vello axilar normal. Mamas normales. Abdomen: Ligero vello en línea alba. Miembros: Ligero vello en pantorrillas. Examen ginecológico: Genitales externos normales. B.S.U. normales. T.V. vagina elástica. Utero normal. Fondos de saco libres. No se palpan masas en los anexos. Citología vaginal: Muestra niveles estrogénicos moderados, en curva ondulatoria, más o menos irregular. Biopsia de Endometrio: "Glándulas endometriales escasas y un pequeño fragmento de endometrio con glándulas pequeñas redondeadas, revestidas por epitelio alto con núcleos alargados, que se disponen perpendicularmente a la luz glandular. Estroma denso hemorrágico. El cuadro histológico sugiere endometrio proliferativo". Se considera que hay estímulo estrogénico adecuado, con oscilaciones, pero sin ritmo. Diagnóstico de Impresión: Ciclos monofásicos. Oligomenorrea. 17 cetoesteroides: $19.3 \mathrm{mgm} .11$ oxicorticosteroides 2.6 mgm. Volumen de orina en 24 h. 2.420 c.c. 
Después del tratamiento con Prednisolona: 17 cetoesteroides: $15 \mathrm{mgm} .11$ oxicorticosteroides: $1.25 \mathrm{mgm}$. Al año siguiente se hicieron nuevas dosificaciones: 17 cetoesteroides: $11.6 \mathrm{mgm}$. 17 hidroxicorticosteroides: $8.3 \mathrm{mgm}$. (Tomando $10 \mathrm{mgm}$. diarios de Prednisolona). I/ ceroesteroides: $15.8 \mathrm{mgm}$. Hidroxicorticosteroides: $6.2 \mathrm{mgm}$. Vol. orina 1.890 c.c. 17 cetoesteroides 6.4 mgm. 11 hidroxicorticosteroides. 3.9 mgm. (Tomando $5 \mathrm{mgm}$. diarios de Prednisolona). 17 cetoesteroides: $17.8 \mathrm{mgm} .11$ hidroxicorticosteroides: $4.9 \mathrm{mgm}$. (Tomando 2.5 mgm. de Prednisolona). Yodoproteinemia: 3.4 gammas\%. Colesterolemia: 2143 mgm. Glicemia: 84 mgm. Ginecografía: Diagnóstico: Ovarios poliquísticos. Cirugía: (Dr. Ricardo Rueda) Resección cuneiforme de ovarios. Anatomía patológica: "Los quistes de ovarios presentan granulosa poco desarrollada. En la corteza se observa buena cantidad de folículos primarios y algunos en maduración. Cortical engrosada. La superficie carece de epitelio germinativo. No se reconocen restos lúteos. Diagnóstico: Ovarios poliquísticos foliculares (Dr. Miguel Mariño). Citología vaginal posterior al tratamiento quirúrgico: Día del ciclo: 9, 14, 19, 24, 29. Ind. Cariopicnótico. 5\%, 50\%, 30\%, 5\%, $20 \%$. 17 cetoesteroides: 15 mgm. 17 hidroxicorticosteroides: $5.5 \mathrm{mgm}$. Yodoproteinemia: 6.2 gammas $\%$. Colesterolemia: $220 \mathrm{mgm} . \%$. Biopsia de endometrio (primer día del ciclo): "Muestra endometrio en fase secretora. Existe hemorragia y glándulas parcialmente destruídas. Diagnóstico: Endometrio menstrual. Yodoproteinemia: 3.8 gammas $\%$. Colesterol: $100 \mathrm{mgm}$. $\%$. Cuadro hemático: Hb. $12.6 \mathrm{mgm}$. Hematocrito $36 \%$. Serología negativa. Espermograma del esposo: Cantidad: $3.5 \mathrm{cc}$. viscosidad normal $\mathrm{pH}$ 7.5 Movilidad 90\%. Recuento: 71 millones. Normales $87 \%$ (Abstención de 6 días). Esta paciente en la actualidad está embarazada.

CASO No 8 - Historia No 661713. Edad 26 áños. Pérdida de peso desde los 17 años. Hipertricosis que apareció en la época de la menarquia a la edad de 16 años. Menarquia inducida. Ciclos posteriores irregulares con fuerte tendencia a períodos largos de amenorreas. 180/3. Antecedentes familiares: Sin importancia. Antecedentes personales: Sin importancia. Antecedentes quirúrgicos: No. Venus de tipo androide. Hipertricosis en cara y miembros inferiores. Examen ginecológico: Clítoris levemente hipertrofiado. Abundante vello vulvo-perineal. Espéculo: Cuello de nulípara. T. R. V. Utero más bien pequeño en Av. y con superficie irregular. Se palpa el ovario izquierdo aumentado de tamaño. Citología vaginal: Indices de picnosis y maduración oscilantes entre $40 \%$ y $70 \%$, lo cual revela actividad estrogénica alta, aunque oscilante. Cromatina sexual positiva. Ginecografía: Positiva para ovarios poliquísticos.
Cirugía: (Dr. Delgadillo) Resección cuneiforme cle ovario derecho y ooforectomía izquierda. El ovario derecho se encontró aumentado de tamaño más o menos $6 \mathrm{~cm}$. por $3 \mathrm{~cm}$. de diámetro. Cápsula gruesa. Numerosos quistes descle $1 \circ 2 \mathrm{~mm}$. a $1 \mathrm{~cm}$. El ovario izquierdo se encontró en regulares condiciones lo que obligó a tomar una conducta radical. Anatomía patológica: (No 67-3356). "... capa cortical engrosada y fibrótica, y por debajo de ésta muchos folículos en diversos estados de maduración y atresia, algunos de ellos quísticos. ...ligera hiperplasia tecal. No hay evidencias de cuerpo amarillo. ...no se observa tumor funcional". Diagnóstico: Ovarios de Stein Leventhal. Ciclos posteriores a la intervención: 28/3.

CASO No 9 - Historia No 489011 - Edad: 22 años. Soltera. Menarquia a los 15 años. Ciclos: 240/8. Examen ginecológico: Normal. Hirsutismo leve. Dismenorrea severa. Acné. T.A. No 120/80. Ginecografía confirmatoria del síndrome de ovarios poliquísticos. Dosificación de 17 cetoesteroides: $8.1 \mathrm{mgm}$ \% . Dosificación de F. S. H.: 65 U. R. Se practicó radiografía de la Silla Turca que fue normal. No se practicó cirugía.

\section{CASOS CONTROLES}

CASO No 1 - Historia No 296395. B. G. Edad: 33 años. Soltera. Menarquia a los 19 años. Ciclos: 365/2. La menarquia fue inducida. Examen ginecológico: Normal. Hirsutismo marcado. Normotensa. Cromatina sexual positiva. Ginecografía normal. Dosificación de 17. cetoesteroides: $22.5 \mathrm{mgm} . \%$. 17 hidroxicorticosteroides: $7.6 \mathrm{mgm}$. \% .

CASO No 2 - Historia No 238619. N. I. Edad: 29 años. Soltera. Hirsutismo marcado. Dismenorrea severa. Normotensa. Examen ginecológico normal. Ginecografía: Hipoplasia de ovario derecho. Dosificación de 17 cetoesteroides. $18.8 \mathrm{mgm}$ \%. Hidroxicorticosteroides: 7.2 mgm.\%. Se practicó biopsia de ovarios que reportó normalidad. Radiografía de Silla Turca normal.

CASO No 3 - Historia No 17916. N. L. Edad: 30 años. Soltera. Menarquia a los 13 años. Ciclos 30/3. Examen ginecológico: Normal. Hirsutismo marcado. Dismenorrea intensa. T.A. 125/70. Ginecografía: Ovario derecho aumentado de tamaño. Moderadamente. Esta paciente anota que anteriormente fue tratada con anabólicos, y que desde entonces aumentó en hirsutismo. Triconeurosis.

CASO No 4 - Historia No 621847. G. P. Edad: 19 años. Soltera. Examen ginecológico: Normal. Hirsutismo marcado. Dismenorrea intensa. Normotensa. Ginecografía: Normal. 17 cetoesteroides: $14.6 \mathrm{mgm} . \%$. 17 hidroxicorticosteroides: $7.3 \mathrm{mgm} . \%$. Se trató con prednisolona. sin resultado. 


\section{COMENTARIOS}

\section{Aspectos clínicos:}

Los casos estudiados fueron de mujeres relativamente jóvenes, ya que las edades oscilaron entre los 18 y 30 años. Todas las pacientes eran solteras excepto una, casada pero sin hijos.

Estos casos ponen de manifiesto la falta de uniformidad en el cuadro clínico de este síndrome (1-2-5). Los primeros signos ya se empiezan a notar en la segunda década, con tendencia a menarquias tardías (5), reglas con ciclos alargados (1-8) y, ocasionalmente dismenorrea; pero solo en la tercera década se hacen manifiestos en especial en referencia a hirsutismo que es el principal motivo de consulta al médico (4). La obesidad y el acné no son signos muy frecuentes (1). En ninguna de las enfermas se halló aumento de tamaño del clítoris por lo cual no se puede hablar de virilismo.

En cuanto a funcionamiento de los ovarios, se puede anotar que en los casos estudiados se encontró una marcada irregularidad de los ciclos menstruales con fuerte tendencia a la oligomenorrea prolongada; aunque hubo dos casos en los cuales los ciclos fueron normales, es decir de 27 y 28 días respectivamente. No se observó amenorrea en ninguno de los casos estudiados. Las menstruaciones fueron irregulares en general en cuanto a cantidad y duración; en algunos casos se observó hipomenorrea y en otros reglas prolongadas.

Como se anotó anteriormente, el hirsutismo adquirió especial importancia en algunos casos en los cuales existía verdadera triconeurosis, especialmente severa en una paciente de 18 años de edad. Todas las pacientes presentaban hirsutismo, en la \\ Rev. Col. Obst. y Ginec.}

mayoría se manifestaba con vello en mejillas, labio superior, mentón y aréolas mamarias. En algunos casos la dismenorrea era muy acentuada.

Con el fin de descartar otras entidades causantes de amenorrea, oligomenorrea e hirsutismo se hicieron estudios clínicos, teniendo en cuenta las cifras tensionales, sin haber hallado alteraciones significativas en éstas.

\section{Exámenes de laboratorio}

La cromatina sexual se practicó en cinco pacientes; en cuatro se halló positiva. Solamente una paciente (Caso № 1) presentó cromatina sexual negativa; el caso se clasificó como pseudo-hermafroditismo masculino, puesto que el sexo gonadal, el sexual y el síquico fueron femeninos y la laparotomía no mostró gónadas masculinas.

En todos los casos se demostró la presencia de ovarios poliquísticos bilaterales, aumentados de tamaño según podía verse en la ginecografía, la cual fue el complemento diagnóstico más útil en su significación y también fué la clave para el diagnóstico correcto en todos los casos. Utilizamos este procedimiento por considerarlo el más adecuado e inocuo ya que solo una paciente presentó dolor en el hombro después de la radiografía.

La ginecografía (6) a nuestra consideración nos dá una visión bastante real del conjunto de útero, ovarios, trompas y ligamentos anchos y también su relación de tamaño, for ma y posición; es posible visualizar claramente el tamaño de los ovarios y observar las irregularidades en la superficie de los mismos, a causa de quistes y en algunos casos engrosamiento de la cortical. 


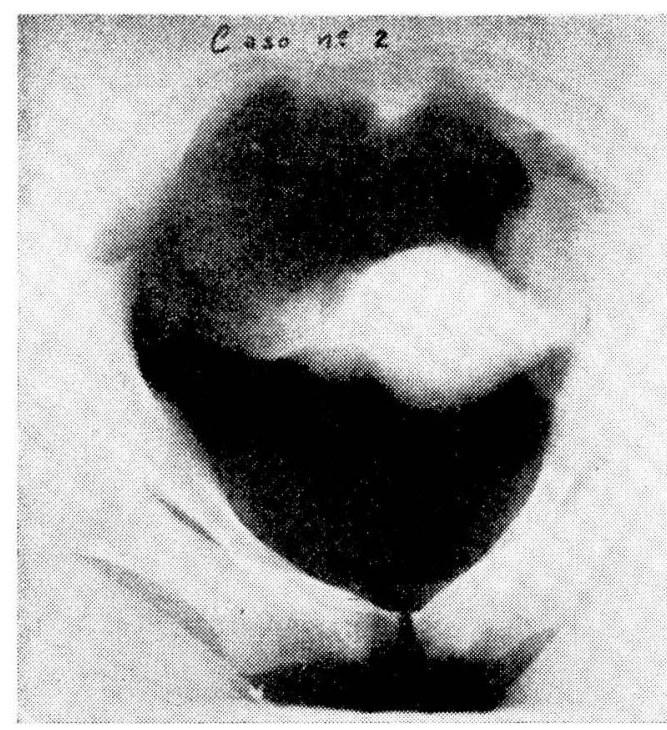

Los estudios correspondientes a los 17 cetoesteroides y los 17 hidroxicorticoesteroides, no mostraron uniformidad, indicando con esto que no es necesariamente un aumento en la producción hormonal lo que se presenta en estos casos. Sin embargo, creemos que tiene más valor la dosificación de los 17 hidroxicorticoesteroides, ya que representa catabolitos de las hormonas suprarrenales.

En cinco casos se practicó resección cuneiforme de ovarios, no siendo posible en los restantes por razones diferentes tales como rechazo de la paciente a la intervención quirúrgica o falta de asistencia de la misma a las consultas.

En esos mismos casos se practicaron estudios anatomopatológicos de los ovarios. En la anatomía patológica llama la atención el hecho de hallar células de la granulosa poco desarrolladas y ausencia de cuerpos lúteos, hallazgos indicadores del tras- torno en la producción hormonal de progesterona, lo cual conduce a un desequilibrio hormonal con ciclos monofásicos e infertilidad. En otros casos se manifiesta la hiperplasia de células tecales, que serían el origen del exceso de producción de hormonas androgénicas que clínicamente se manifiestan con hipertricosis.

Otro de los mecanismos etiopatogénicos que ha sido descrito, es el relativo a un defecto enzimático congénito. La falta de una enzima (2) la 3-beta-ol-dehidrogenasa del ovario, que causaría un trastorno en la síntesis final de los estrógenos y la consiguiente acumulación de sustancias con acción androgénica.

También por otros mecanismos se presentaría una pérdida del equilibrio hormonal, desplazado hacia el lado de los andrógenos con la consiguiente sintomatología clínica.

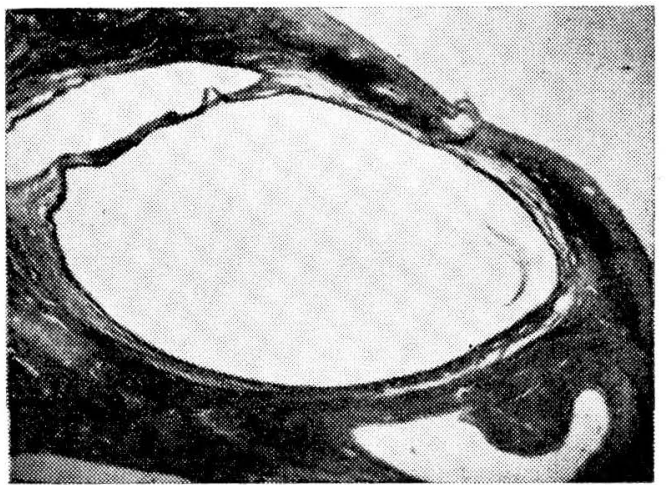

En dos de los pacientes se practicaron dosificaciones de 17 cetoesteroides después del tratamiento quirúrgico, observándose una disminución de los valores encontrados inicialmente.

En algunas pacientes se practicó dosificación de F. S. H., pero los va- 
lores fueron variables. En una paciente se tomó radiografía de la Silla Turca, la cual fue normal.

Se intentaron tratamientos con Prednisolona en tres pacientes, y con anovulatorios en una paciente. Ambos tratamientos fueron ineficaces.

Solamente una paciente pudo controlarse después de la resección cuneiforme de ovarios, $y$ en ella se observó una completa normalidad en los ciclos menstruales; antes de la intervención presentaba ciclos de 240/3; después de la intervención presentó ciclos de 28/3. Esta paciente en la actualidad cursa su primer embarazo normal.

\section{Casos controles}

Se estudiaron cuatro casos controles que presentaban una sintomatología muy parecida a los anteriores, por lo cual se sometieron a los mismos procedimientos de diagnóstico.

En este grupo de pacientes las edades correspondieron entre los $19 \mathrm{y}$ 30 años. Todas las pacientes eran solteras.

La menarquia fue sensiblemente normal. Solamente en un caso se observó una menarquia tardía, a los 19 años, la cual fué inducida. En esta misma paciente los ciclos menstruales eran prolongados, hasta de 265/2, con tendencia a la hipomenorrea. Las demás pacientes presentaron ciclos relativamente normales.

En estos controles el síntoma predominante fue el hirsutismo, que en todos los casos fue bastante intenso, $y$ como en los anteriores, se caracterizaba por presencia de vello en la cara, aréolas mamarias y miembros.
Ninguna paciente presentó acné. Los datos tensionales fueron normales.

Solamente en una paciente se hizo determinación de la cromatina sexual, la cual fue normal.

A todas las pacientes se les practicó Ginecografía. Dos fueron normales. En una se observó hipoplasia de ovario derecho. En la otra se observó aumento de tamaño del ovario derecho.

Las cifras de 17 cetoesteroides y 11 hidroxicorticoesteroides fueron normales. Una de las pacientes anotó como hecho de algún valor, el haber tomado anabólicos y que desde entonces le apareció vello.

Se utilizaron tratamientos a base de Prednisolona, con resultados negativos. En una paciente se practicó radiografía de la silla turca, que fue normal.

\section{RESUMEN}

Se presenta el estudio en nueve pacientes con diagnóstico de Ovarios Poliquísticos de Stein Leventhal.

En cinco de ellas se efectuó cirugía y anatomía patológica; los casos fueron estudiados con Ginecografía y Análisis hormonales.

Se pone de manifiesto el valor de las alteraciones menstruales y la hipertricosis en el aspecto clínico y se analizan los resultados de los hallazgos hormonales $y$ anatomopatológicos.

Se concluye sobre la importancia de la Ginecografía en el síndrome que nos ocupa y sobre los resultados de la terapéutica empleada.

Estudios similares se realizaron en cuatro casos control, que presentaban sintomatología similar a los casos de Stein Leventhal. 
Vol. $\mathrm{Xix}$

$\mathrm{N}^{0} 3$

OBSERVACIONES PERSONALES SOBRE

EL SINDROME DE OVARIOS POLIQUISTICOS

\section{BIBLIOGRAFIA}

1 INGERSOLL F. M., The policystic-ovary síndrome. Clin. Obst. and Gin., Vol. 4:807, 1961.

2 SALCEDO, C. A. y GOMEZ R. A. Aspectos clínicos y bioquímicos del síndrome de ovarios poliquísticos de Stein-Leventhal. Rev. Col. Obs. y Gin., Vol. XVIII, № $1: 21$, 1967.

3 GOUBERT L. C. A. Tumores del ovario. Rev. Col. Obs. y Gin., Vol. XVI. Nọ 3, 237, 1965.

4 RAVERA, J. J. Síndrome de Stein-Leventhal. Anales Fac. Med. Montev., 43 No 5-6, 207, 1958.
5 RILEY G. M. Disfunciones menstruales. Endocr. Ginecol., 97, 1960. M-xico.

6 UFER, J., Amenorrea con ovario poliquístico. Hormonoter. En Gin-Obs. 94, 1965. Madrid.

7 TELINDE, R. W. Tumores del Ovario. Gin. Operat. 701, 1956.

8 BOTELLA, LL. J. Síndromes ováricos. Endocr. de la mujer. 546, 1961. Barcelona.

9 NOVAK, N., Non-neoplastic Cysts of Ovary. Gin. and Obs. Pathology. 340, 1958. Philadelphia.

10 KOSS L. G. Cytologic Evaluation of endocrine disorders. Diagnostic Cytology. 58, 1961. Philadelphia. 